\title{
Primary drug resistance of mycobacterium tuberculosis in Shandong, China, 2004- 2018
}

Wan-mei Song ${ }^{1,2+} \mathbb{D}$, Yi-fan Li ${ }^{1 \dagger}$, Xiao-bin Ma ${ }^{1}$, Jin-yue Liư ${ }^{3}$, Ning-ning Tao ${ }^{4}$, Yao Liu', Qian-yun Zhang ${ }^{1,2}$, Ting-ting $X u^{1}$, Shi-jin $\mathrm{Li}^{1,2}$, Chun-Bao $\mathrm{Yu}^{5}$, Lei Gao ${ }^{6}$, Liang-liang Cui ${ }^{7,8}$ and Huai-chen $\mathrm{Li}^{1,9^{*}}$

\begin{abstract}
Background: Primary drug-resistant tuberculosis (DR-TB) has contributed to a significant health and economic burden on a global scale, especially in China. we sought to estimate epidemiological characteristics of primary DR-TB in China from 2004 to 2018.

Methods: Eleven thousand four hundred sixty-seven newly diagnosed and 1981 retreated TB cases with drug susceptibility data were included. Chi-Square test for trends, linear regression, a joinpoint regression model and temporal trend in proportions of the different resistance patterns were carried out.

Results: The proportion of primary DR-TB and mono-resistant TB (MR-TB) in China had reduced by more than $12 \%$ since 2004 , and were $21.38 \%, 13.35 \%$ in 2018 respectively. Among primary DR-TB cases $(2173,18.95 \%)$, the percentage of multiresistant TB (MDR-TB, from 5.41 to 17.46\%), male (from 77.03 to 84.13\%), cavity (from 13.51 to 43.92\%), rifampicin(RFP)-resistant TB (from 8.11 to 26.98\%), streptomycin(SM)-resistant TB (from 50.00 to $71.43 \%$ ) increased significantly $(P<0.05)$. On the contrary, the proportion of female, non-cavity, isoniazide(INH)-resistant TB (from 55.41 to $48.15 \%$ ) and MR-TB (from 82.43 to $62.43 \%$ ) decreased significant $(P<0.05$ ). The primary drug resistance rate among female, cavity, smoking, drinking, 15 to 44 year-old TB subgroups increased by $0.16,6.24,20.95,158.85$, $31.49 \%$, respectively. The percentage of primary DR-TB, RFP-resistant TB dropped significantly during 2004-2007 in Joinpoint regression model.

Conclusion: The total rate of drug resistance among new TB cases showed a downward trend in Shandong, China, from 2004 to 2018. Primary drug resistance patterns were shifting from female, non-cavity, INH-resistant TB, and MR-TB groups to male, cavity, RFP/SM-resistant TB, and MDR-TB groups. Considering the rising drug resistance rate among some special population, future control of primary DR-TB in China may require an increased focus on female, cavity, smoking, drinking, or 15 to 44 year-old TB subgroups.
\end{abstract}

Keywords: Tuberculosis, Primary drug resistance, Epidemiology, Risk factors, Joinpoint regression model

\section{Background}

Drug resistance of mycobacterium tuberculosis (MTB), especially multidrug resistant tuberculosis (MDR-TB, defined as resistance to at least rifampin and isoniazid) has contributed to a significant health and economic

\footnotetext{
* Correspondence: lihuaichen@163.com

+Wan-mei Song and Yi-fan Li contributed equally to this work.

${ }^{1}$ Department of Respiratory Medicine, Shandong Provincial Hospital Affiliated

to Shandong University, Jinan 250021, Shandong, China

${ }^{9}$ Shandong University of Traditional Chinese Medicine, Jinan 250355,

Shandong, China

Full list of author information is available at the end of the article
}

burden on a global scale [1-3]. Drug-resistant tuberculosis (DR-TB) can be transmitted (primary resistance, refers to the infection with drug-resistant MTB) or develop during the course of treatment (secondary or acquired resistance) [3]. Plenty of researches [4-6] had revealed that the majority of DR-TB were primary instead of acquired drug resistance, in other words, the main mechanism of drug resistance in TB was the transmission of drug-resistant MTB strains from the existing TB patients rather than mismanagement of previous treatment episode. For instance, a molecular epidemiological

(C) The Author(s). 2019 Open Access This article is distributed under the terms of the Creative Commons Attribution 4.0 International License (http://creativecommons.org/licenses/by/4.0/), which permits unrestricted use, distribution, and 
study on DR-TB strains in Shanghai using highly discriminatory whole genome sequencing (WGS) found that more than $73 \%$ of MDR-TB cases were caused by primary transmission $[5,6]$.

According to the World Health Organization (WHO) Global TB report 2018, an estimated 10 million people developed TB in 2017, of whom 3.5\% of newly diagnosed and $18 \%$ of retreated $\mathrm{TB}$ cases were MDR-TB, and China had the world's second largest number of $\mathrm{TB}$ cases and MDR/rifampicin-resistant $\mathrm{TB}(\mathrm{RR}-\mathrm{TB})$, comprising around 9 and $13 \%$ of the world total, respectively, behind only India (TB cases, 27\%; MDR/RR-TB, 24\%) [7]. Based on a national survey of drug-resistant tuberculosis (DR-TB) in China, there were 869,092 new TB cases and 54,216 previously treated TB cases in 2010, and the rate of primary and acquired MDR-TB were 5.7\% (95\% confidence interval [CI], 4.5-7.0) and 25.6\% (95\% CI, 21.5-29.8), respectively [4]. Great progress in TB control and prevention had been made in China since the implement of DOTS strategy in 1990s, and prevalence rate of TB reduced $3.4 \%$ per year [8]. However, previous studies reported that the rate of total DR-TB remained a high level, and the overall MDR rate was $6.2 \%$ in Shandong during 2007-2014, overall rifampin (RFP) resistance and rifampin monoresistance (RMR) increased at a yearly rate of 0.2 and $0.1 \%$, respectively [9].

Understanding the burden of primary DR-TB and the factors associated with its transmission may help determine the high-risk population for drug-resistant MTB infection and develop measures for preventing transmission. Moreover, investigating the current epidemiological status of primary DR-TB in China also help to assess the effectiveness of existing TB interventions and provide guidance for TB control in future. However, few studies $[4,10]$ had focused on the epidemic of primary DR-TB in China. To evaluate the trend, high risk factors and epidemiological characteristics of primary DR-TB in China from 2004 to 2018, we collected 11,467 new TB cases with drug susceptibility test (DST) results for statistical analysis by applying a join-point regression model and chi-square regression for trend, and DR-TB groups were stratified by drug-resistant profiles, age, sex, smoking, drinking and cavity for further analysis.

\section{Materials and methods}

\section{Ethics statement}

The protocols applied in this study were approved by the Ethics Committee of Shandong Provincial Hospital, affiliated with Shandong University ( $\mathrm{SPH}$ ) and and the Ethic Committee of Shandong Provincial Chest Hospital $(\mathrm{SPCH})$, China. Before analysis, patient records were anonymized and deidentified.

\section{Setting}

This study was carried out in Shandong, a coastal province of the East China region. Shandong consisted of 17 municipalities and 140 counties (districts) with 95.8 million inhabitants at the 2010 Census. It is located at $36^{\circ} 24^{\prime} \mathrm{N}$ latitude $118^{\circ} 24^{\prime} \mathrm{E}$ longitude with an area of 157 , $100 \mathrm{~km}^{2}$. Shandong has emerged as one of the most populous and most affluent provinces in China since the late nineteenth century.

\section{Study population and data collection}

A total of 11,467 newly diagnosed and 1981 retreated MTB cases were collected from $36 \mathrm{~TB}$ prevention and control institutions of Shandong Province, China, Jan 1, 2004 to Dec 31, 2018. Two province-level hospitals (SPH and $\mathrm{SPCH}), 13$ municipal-level and 21 county-level local health departments were involved in the surveillance of DR-TB from 2004 to 2018. All MTB cases enrolled in our study were consecutive culture-confirmed and finished DST for first line anti-TB drugs. Demographic and clinical characteristics on age, sex, drinking, smoking, cavity, treatment history and extra-pulmonary TB were available. Smoker (or drinker) refers to those who satisfy at least one of the following two conditions: i) smoking (or drinking) for 6 months or above; ii) those who was still smokin$\mathrm{g}$ (or drinking) or had stopped smoking (or drinking) for less than 6 months before TB diagnosis. Non-smoker and non-drinker were defined as the person who had never smoked or never drunk, respectively.

Bacteriologic examinations and drug susceptibility testing Each surveillance site collected two sputum samples from all eligible patients, and then sent all sputum samples to the TB Reference Laboratory of SPCH for further examination including bacteriologic culture, DST, and species identification. Isolates were inoculated into tubes of acidified Löwenstein-Jensen (LJ) medium after conventional pretreatment process and cultured at $37^{\circ} \mathrm{C}$ [4]. Cultures with growing colonies were sent for further identification and DST. According to previous published protocol [11], standard traditional biochemical testings such as P-nitrobenzoic acid, 2-thiophene carboxylic acid hydrazide testing and $16 \mathrm{~S}$ rRNA gene sequence analysis (MicroSeq ID Microbial Indentification Software(version 2.0); Applied Biosystems, Foster City, CA, USA) [12] were used to differentiate $M$. tuberculosis from other Mycobacteria spp.. DST of $M$. tuberculosis were performed using absolute concentration method on L-J media, and all procedures were carried out in accordance with the guideline of WHO [13]. The concentration of four first-line anti-TB drugs were as follows: $0.2 \mu \mathrm{g} / \mathrm{mL}$ (isoniazid, INH), $40 \mu \mathrm{g} / \mathrm{mL}$ (rifampin, RFP), $10 \mu \mathrm{g} / \mathrm{mL}$ (streptomycin, SM), $2 \mu \mathrm{g} / \mathrm{mL}$ (ethambutol, EMB). DST 
of ethionamide, fluoroquinolone, kanamycin and pyrazinamide were not routinely performed.

\section{Quality control}

Two professionally trained investigators were independently responsible for quality assessment and data extraction; and all laboratories involved in our study regularly accepted external quality assessment of Superior TB National Reference laboratory in SPCH.

\section{Definitions}

Drug-resistant tuberculosis (DR-TB) are classified as having acquired or primary drug resistance on the basis of a history of previous treatment [13].

Mono-resistance (MR) refers to resistance to one firstline anti-TB drug only [14].

Polydrug resistance (PDR) refers to resistance to more than one first-line anti-TB drug, other than both isoniazid and rifampicin [14].

Multidrug resistance (MDR) refers to resistance to at least both isoniazid and rifampicin [14].

\section{Statistical analysis}

Categorical variables including age $(0-14,15-44,45-64$, $65+$ ), sex(male or female), drinking (yes/no/unknown), smoking (yes/no/unknown), cavity (yes/no/unknown), patients type (extra-pulmonary TB/pulmonary $\mathrm{TB}$ ) of new and relapse TB cases were calculated as counts and proportions, respectively. In addition, odds ratios (ORs) and 95\% CIs for the comparisons of these characteristics were estimated between newly diagnosed susceptible and DRTB cases, retreated susceptible and DR-TB cases, new and relapse TB cases, primary and acquired DR-TB cases. Differences in drug susceptibility profiles between primary DR M. tuberculosis and acquired drug-resistant M. tuberculosis isolates were analyzed using Pearson Chi-square test, and $p$-value $<0.05$ was considered statistically significant. Chi-square test for trends and linear regression in line charts and stacked bar charts were used to assess the changes and temporal trend in quantity and proportions of the different resistance patterns among total DR-TB cases from 2004 to 2018.

We calculated total or annual primary drug resistance rate of tuberculosis as the number of annual primary DR-TB cases divided by the population of newly diagnosed TB cases. The overall rate and the annual rate stratified by age groups, sex, drinking (yes/no/unknown), smoking (yes/no/unknown), cavity (yes/no/unknown) and type (extra-pulmonary TB/pulmonary $\mathrm{TB}$ ) were estimated as well. Indicators including annual percent changes (APCs) and average annual percent changes (AAPCs) were calculated from 2004 to 2018 inclusive on the basis of a joinpoint regression model. Each segment described a short-term trend (APC). Long-term trends over the entire study period are AAPCs and were estimated as the weighted average of the short-term APCs, with the weights equal to the length of the short-term line segment [15]. Two-sided $t$-tests at $p<0.05$ were used to test whether AAPCs and APCs were statistically significantly different from zero; $95 \%$ CI for each segment were calculated. If the AAPC was within one segment, the $\mathrm{t}$-distribution was used. Otherwise, the normal (z) distribution was used [15-17]. A non-significant ( $p \geq$ $0.05)$ APC was described as stable while a significant $(p<0.05)$ positive or negative APC was termed as increase or decrease. All analyses were carried out using SPSS software (version 20.0) and the Joinpoint Regression Software (version 4.3.1.0).

\section{Results}

\section{Patients' characteristics}

Table 1 illustrates the baseline characteristics of the study participants. A total of 11,467 new and 1981 relapse TB cases were reported during the period from Jan 1, 2004 to Dec 31, 2018 in Shandong, China, of which 2173 (18.95\%) and $505(23.24 \%)$ were drug-resistant TB cases, respectively, $P<0.01$. Among these primary DR-TB cases, there were more males $(83.06 \%$ vs $16.94 \%)$, non-smokers ( $40.73 \%$ vs $13.90 \%)$, non-drinkers ( $42.84 \%$ vs $11.50 \%$ ), pulmonary TB $(99.68 \%$ vs $0.32 \%)$. In addition, most primary DR-TB cases $(2168,99.77 \%)$ were from three age groups: $15-44$ years $(930,42.80 \%), 45-64$ years $(728,33.50 \%)$, > 65 years $(486,22.37 \%)$. The distribution of age, sex, smoking, et al. among acquired DR-TB cases were similar to primary DR-TB cases (Table 1).

Compared with newly diagnosed susceptible TB cases, primary DR-TB cases were more likely to be female (OR: 1.166, 95\%CI:1.031-1.320) and less likely to be aged more than 65(OR: 0.840, 95\%CI:0.745-0.948). Among retreated TB cases, factors associated with acquired resistance were baseline cavitary diseases (OR: 1.544, 95\%CI:1.24-1.924) and $>65$ years (OR:0.684, 95\%CI:0.521-0.900). New TB were less likely to be aged 45-64 years old (OR:0.84, 95\%CI: 0.750-0.942) or $>65$ years old (OR:0.851, 95\%CI: $0.753-0.962)$ when compared with relapse TB cases. Moreover, patients with pulmonary $\mathrm{TB}$ had a statistically significant higher risk of primary drug resistance (vs acquired drug resistance) than those with extra-pulmonary TB (OR: 4.349, 95\%CI:1.519-12.456) (Table 1).

\section{Resistance patterns}

Among 11,467 newly diagnosed and 1981 retreated TB clinical isolates, the number and proportion of any resistance to first-line drugs including INH, RFP, EMB, SM were $1232(10.74 \%)$ and 333(16.81\%), 508(4.43\%) and 223(11.26\%), 174(1.52\%) and 68(3.43\%), 1482(12.92\%) and $329(16.61 \%)$, respectively; new TB cases had a lower rate 


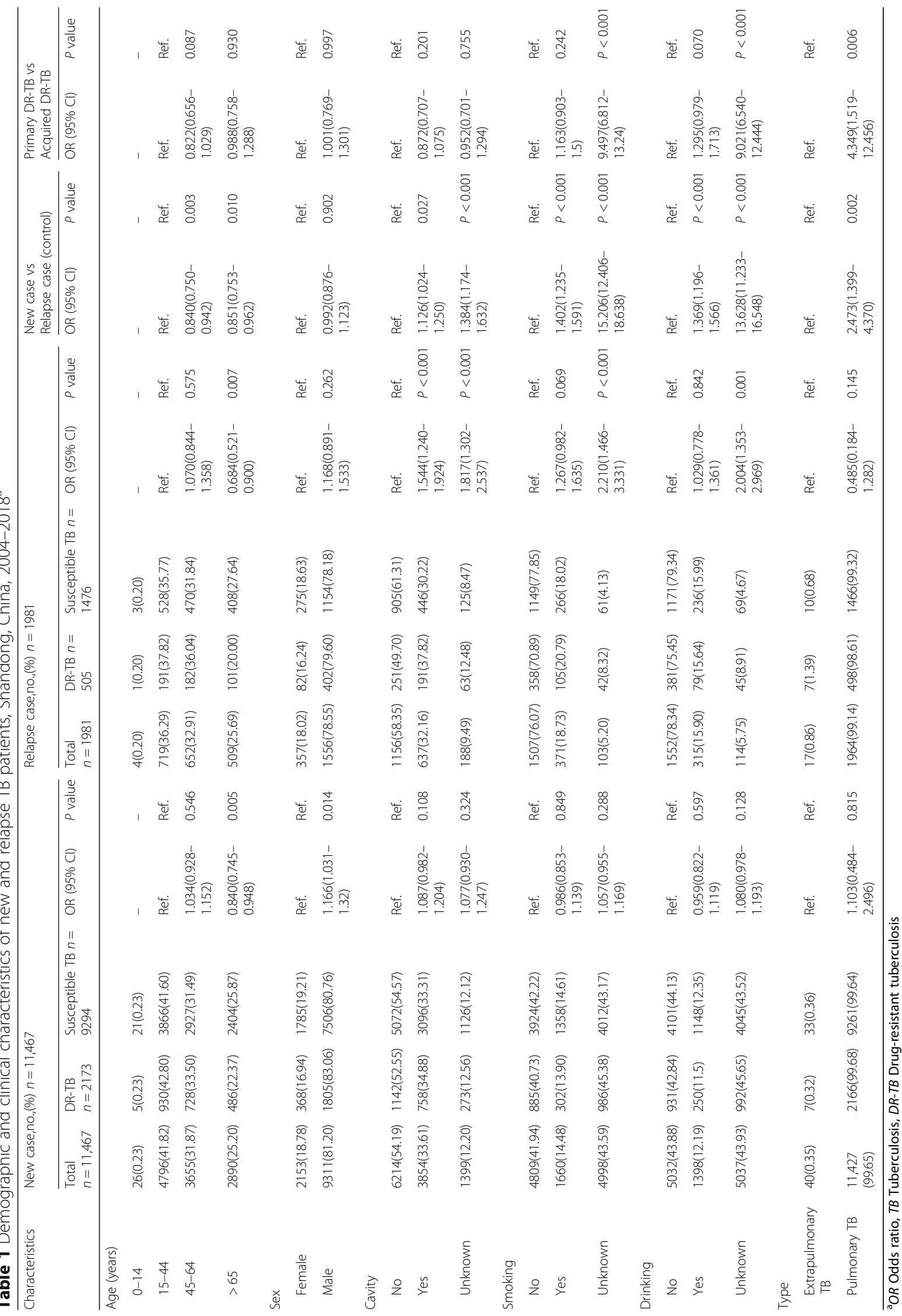


of DR-TB(18.95\% vs $23.24 \%, P<0.01)$, MDR-TB(3.19\% vs $8.43 \%, P<0.01)$, PDR-TB( $4.12 \%$ vs $5.55 \%, P<0.01)$ than relapse TB cases, but almost the same in MR-TB $(11.56 \%$ vs $11.36 \%, P>0.05)$. Of 1326 primary MR-TB cases, SM was associated with the highest rate of resistance $(6.41 \%$, $758)$, followed by INH $(3.88 \%, 445)$, RFP $(0.78 \%, 90)$, and $\operatorname{EMB}(0.17 \%, 20)$. There were four main types of primary MDR-TB, for instance, MDR1 (INH + RFP), MDR2 $(\mathrm{INH}+\mathrm{RFP}+\mathrm{EMB}), \quad \mathrm{MDR} 3 \quad(\mathrm{INH}+\mathrm{RFP}+\mathrm{EMB}+\mathrm{SM})$, and MDR4 (INH + RFP + SM), which accounted for $0.71 \%(81), 0.13 \%(15), 0.72 \%(83), 1.45 \%(166)$ respectively. Similarly, primary PDR-TB mainly consisted of PDR1 (INH + EMB, 0.12\%, 14), PDR2 (INH + SM, 3.41\%, 391), PDR3 (RFP + EMB, 0.06, 7), PDR4 (RFP + SM, 0.33\%, 38), PDR5 (INH + EMB + SM, 0.14\%, 16) (Table 2).

\section{Disparity in the proportion of primary DR-TB subgroups}

As shown in Figs. 1a-f and 2, primary DR-TB cases was sub-divided into different subgroups according to the types of drug resistance (MR-TB/MDR-TB/PDR-TB, INH/RFP/
SM/EMB-resistance), age (0-14, 15-44, 45-64, 65+), sex (male or female), drinking history (yes/no/unknown), smoking history (yes/no/unknown), cavity (yes/no/unknown), and the amount and proportions of each subgroup among the total primary DR-TB cases varied every year since 2004 to 2018. Among all primary DR-TB cases, the percentage of MDR-TB ( $\left[R^{2}=0.2034\right]$ from 5.41 to $17.46 \%$; $\chi 2$ test for trends: $\chi 2=5.376, P=0.020)$, males $\left(\left[R^{2}=\right.\right.$ 0.4834 from 77.03 to $84.13 \%$; $\chi 2$ test for trends: $\chi^{2}=$ $12.570, P<0.001)$, cavity $\left(\left[R^{2}=0.7022\right]\right.$ from 13.51 to 43.92\%; $\chi 2$ test for trends: $X 2=120.53, P<0.001)$, RFPresistance $\left(\left[R^{2}=0.5355\right]\right.$ from 8.11 to $26.98 \% ; \chi 2$ test for trends: $\chi 2=16.785, P<0.001)$, SM-resistance $\left(\left[R^{2}=0.5365\right]\right.$ from 50.00 to $71.43 \%$; $\chi 2$ test for trends: $\chi 2=22.076, P<$ 0.001 ) increased significantly from 2004 to 2018 . On the contrary, the proportion of females, non-cavity, INH $\left(\left[R^{2}=\right.\right.$ $0.4269]$ from 55.41 to $48.15 \% ; X^{2}$ test for trends: $\chi^{2}=$ $14.725, P<0.001)$ and MR-TB $\left(\left[R^{2}=0.178\right]\right.$ from 82.43 to 62.43\%; $\chi 2$ test for trends: $X 2=6.287, P=0.012)$ decreased significantly $(P<0.05)$ (Table 5 in Appendix).

Table 2 Primary and acquired drug resistance profiles of Mycobacterium tuberculosis, Shandong, China, 2004-2018

\begin{tabular}{|c|c|c|c|}
\hline Drug resistance & New case, no., (\%) $n=11,467$ & Relapse case, no., (\%) $n=1981$ & $P$ value \\
\hline$\overline{\mathrm{DR}-\mathrm{TB}}$ & $2173(18.95)$ & $505(23.24)$ & $P<0.001$ \\
\hline \multicolumn{4}{|l|}{ Any resistance to first-line drugs } \\
\hline $\mathrm{INH}$ & $1232(10.74)$ & $333(16.81)$ & $P<0.001$ \\
\hline RIF & $508(4.43)$ & 223(11.26) & $P<0.001$ \\
\hline$E M B$ & $174(1.52)$ & $68(3.43)$ & $P<0.001$ \\
\hline SM & $1482(12.92)$ & $329(16.61)$ & $P<0.001$ \\
\hline MR-TB (Total) & 1326(11.56) & $225(11.36)$ & 0.791 \\
\hline $\mathrm{INH}$ & $445(3.88)$ & $82(4.14)$ & 0.584 \\
\hline RIF & $90(0.78)$ & $29(1.46)$ & 0.003 \\
\hline EMB & 20(0.17) & $9(0.45)$ & 0.013 \\
\hline SM & $758(6.61)$ & $99(5.00)$ & 0.007 \\
\hline Others & 13(0.11) & $6(0.30)$ & 0.038 \\
\hline MDR-TB (Total) & 366(3.19) & $167(8.43)$ & $P<0.001$ \\
\hline MDR1:INH + RFP & $81(0.71)$ & $37(1.87)$ & $P<0.001$ \\
\hline MDR2:INH + RFP + EMB & 15(0.13) & $6(0.30)$ & 0.073 \\
\hline$M D R 3: I N H+R F P+E M B+S M$ & 83(0.72) & $33(1.67)$ & $P<0.001$ \\
\hline MDR4:INH + RFP + SM & $166(1.45)$ & $76(3.84)$ & $P<0.001$ \\
\hline Others & $21(0.18)$ & $15(0.76)$ & $P<0.001$ \\
\hline PDR-TB & $473(4.12)$ & $110(5.55)$ & 0.004 \\
\hline PDR1:INH + EMB & $14(0.12)$ & $1(0.05)$ & 0.713 \\
\hline PDR2:INH + SM & $391(3.41)$ & 77(3.89) & 0.285 \\
\hline PDR3:RFP + EMB & $7(0.06)$ & $3(0.15)$ & 0.173 \\
\hline PDR4:RFP + SM & 38(0.33) & 19(0.96) & $P<0.001$ \\
\hline PDR5:INH + EMB + SM & $16(0.14)$ & $5(0.25)$ & 0.240 \\
\hline Others & $7(0.06)$ & $5(0.25)$ & 0.008 \\
\hline
\end{tabular}

${ }^{a} E M B$ ethambutol, INH Isoniazid, RFP Rifampin, SM Streptomycin, TB Tuberculosis, DR-TB Drug-resistant tuberculosis, MR-TB Mono-resistant tuberculosis, MDR-TB Multi-resistant tuberculosis, $P D R-T B$ Polydrug resistant tuberculosis 

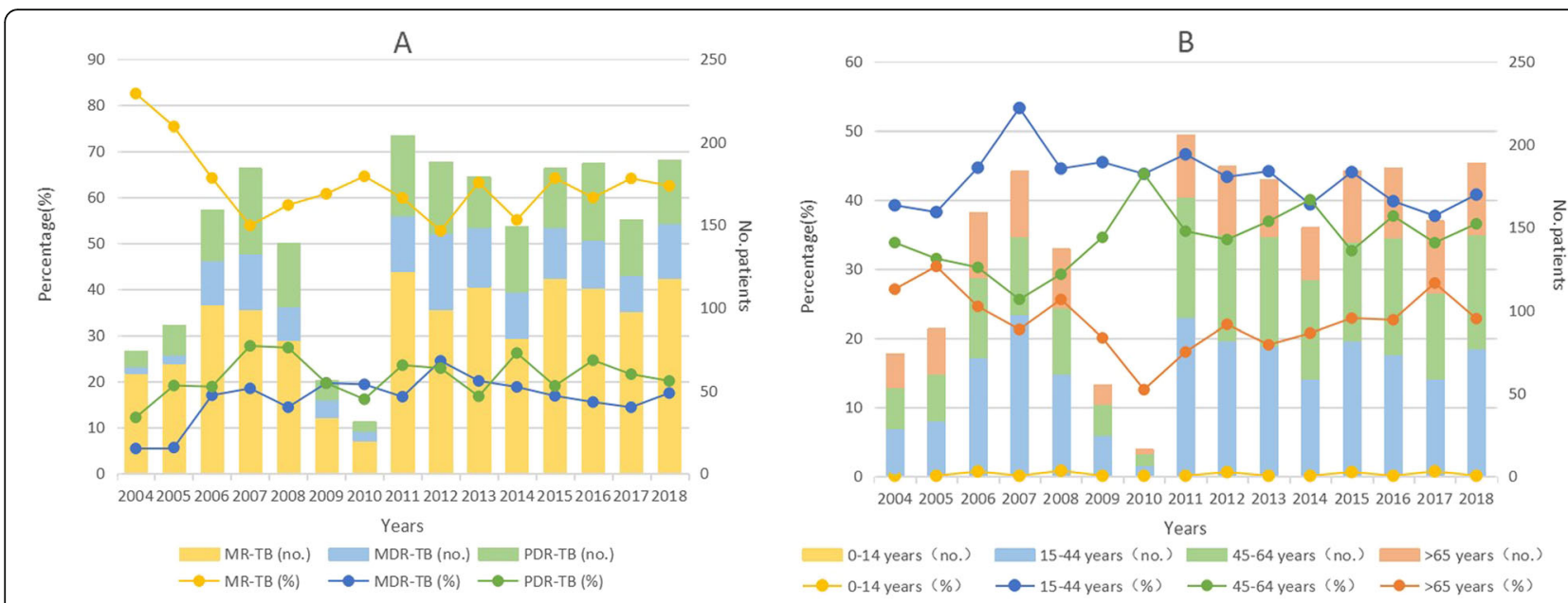

C
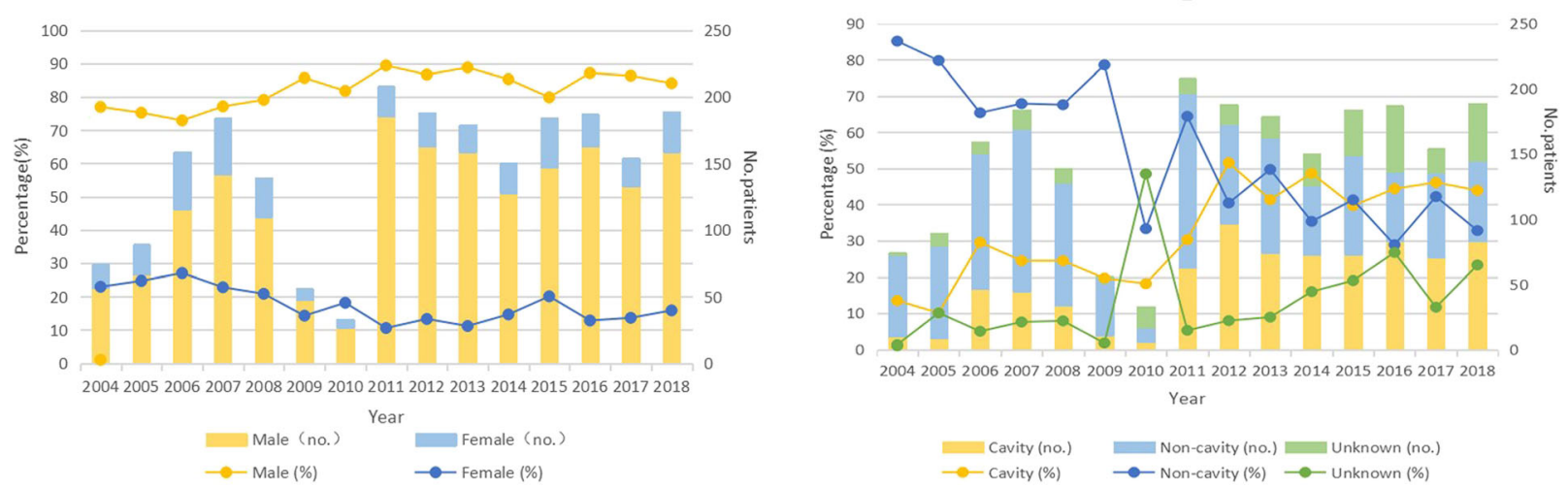

Year

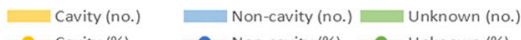

$\rightarrow$ Cavity (\%) $\rightarrow$ Non-cavity (\%) $\rightarrow$ Unknown (\%)

$\mathrm{E}$

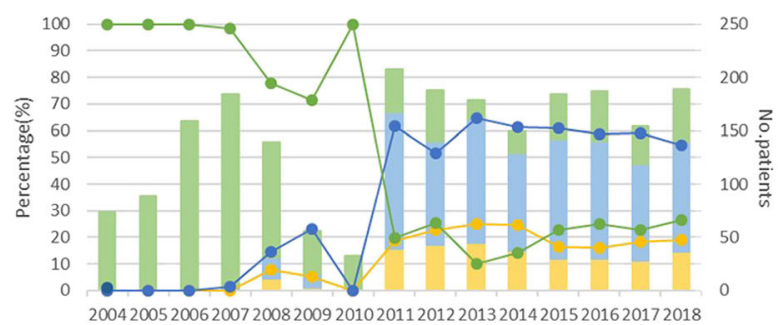

$\mathrm{F}$

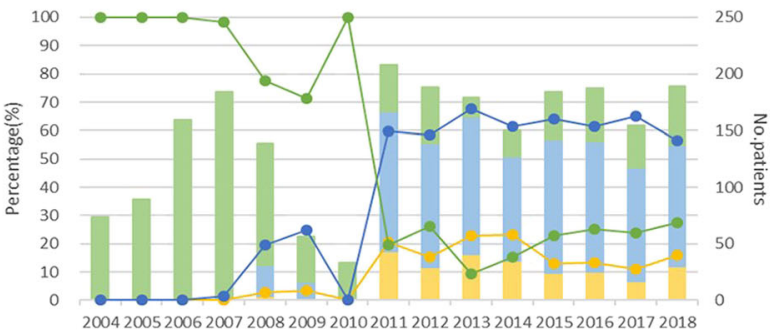

Year

Smoking (no.) Non-smoking (no.) Unknown (no.)

$\longrightarrow$ Smoking (\%) $\rightarrow$ Non-smoking (\%) $\longrightarrow$ - Unknown (\%)

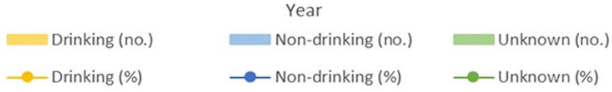

Fig. 1 Trends for the quantity and proportions of different subgroups among total primary DR-TB cases, Shandong, China, 2004-2018*a a Trends for MR-TB, MDR-TB, PDR-TB among total primary cases of TB; $\mathbf{b}$ Trends for primary DR-TB cases of different age (0-14, 15-44, 45-64, 65+); $\mathbf{c}$ Trends for primary DR-TB cases of different sex(male or female); $\mathbf{d}$ Trends for primary DR-TB cases with or without cavity; e Trends for primary DRTB cases with or without smoking history; $\mathbf{f}$ Trends for primary DR-TB cases with or without drinking history; The proportions of each subgroups were calculated as follows: (the quantity of each subgroups/ the quantity of total primary DR-TB subgroups in the same year) ${ }^{*} 100 \%$. The $X^{2}$ and linear regression results are shown in Table 5 in Appendix. TB, tuberculosis; DR-TB, drug-resistant tuberculosis; MR-TB, mono-resistant tuberculosis; MDR-TB, multi-resistant tuberculosis; PDR-TB, polydrug resistant tuberculosis. EMB, ethambutol; INH, isoniazid; RFP, rifampin; SM, streptomycin

Total and annul primary drug resistance rate

Table 3 illustrates the overall and annul primary drug resistance rate of various TB subgroups from 2004 to 2018. During the study period, the primary drug resistance rate among female, cavity, smoking, drinking, 15 to 44 year-old TB subgroups increased by $0.16,6.24$, $20.95,158.85,31.49 \%$, and varied from 20.24 to $20.27 \%$, from 20.83 to $22.13 \%$, from 21.57 to $26.09 \%$, from 10.26 


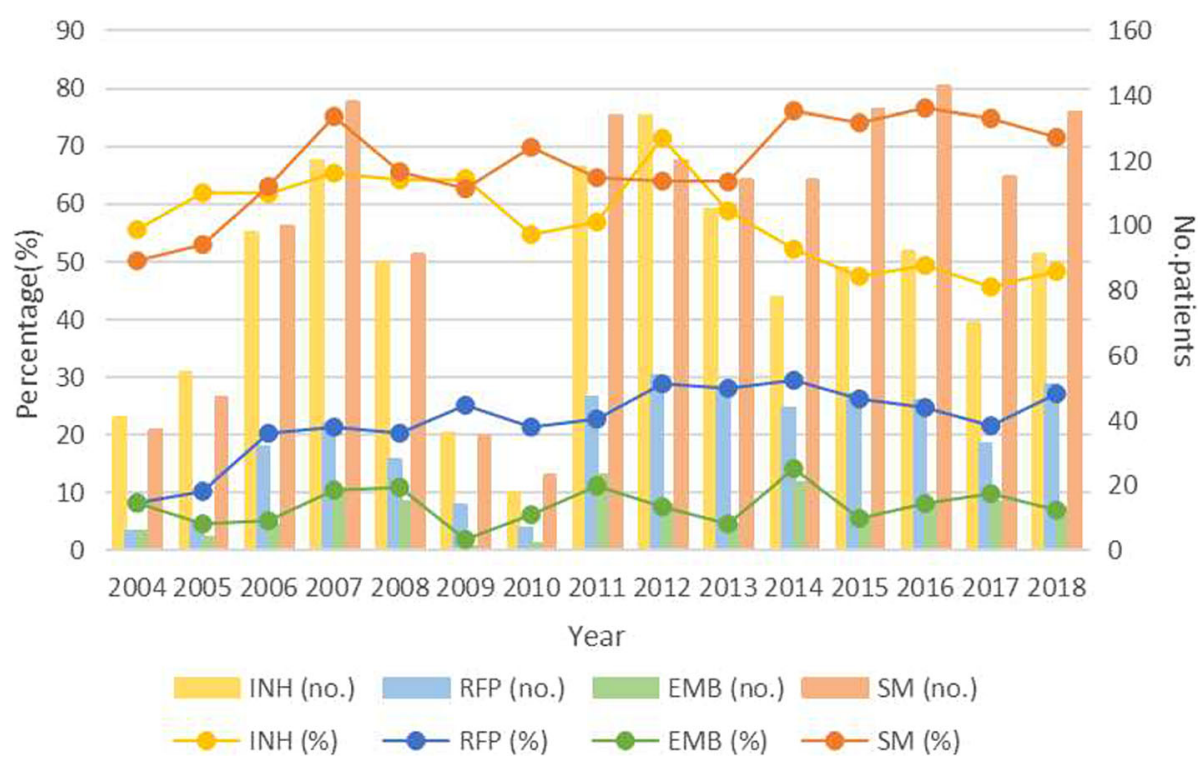

Fig. 2 Overall first-line drug resistance for INH, RIF, EMB, and SM in primary cases of tuberculosis in Shandong China, 2004-2018. The proportions of INH-, RIF-, EMB-, and SM-resistance were calculated as follows: (the quantity of each subgroups/ the quantity of total primary DR-TB subgroups in the same year)*100\%. The $x^{2}$ and linear regression results are shown in Table 5 in Appendix. EMB, ethambutol; INH, isoniazid; RFP, rifampin; SM, streptomycin

to $26.55 \%, 18.59$ to $24.44 \%$, respectively; meanwhile the rate of DR-TB among male, non-cavity, non-smoking, non-drinking, 45 to 64 year-old, > 65 year-old TB subgroups reduced by $16.62,26.34,15.79,22.18,35.01$, $36.66 \%$ respectively, decreasing from 20.24 to $20.27 \%$, 24.90 to $18.34 \%, 21.57$ to $26.09 \%, 25.00$ to $19.45 \%, 32.47$ to $21.10 \%$, and 28.17 to $17.84 \%$, respectively. In addition, the rate of MDR-TB, PDR-TB, RFP-resistant TB, and SM-resistant TB among new TB cases increased from 1.32 to $3.73 \%, 2.96$ to $4.30 \%, 1.97$ to $5.77 \%$, and 12.17 to $15.27 \%$, with a percentage change of $183.71,45.20$, 192.31, 25.47\%, respectively; the rates of MR-TB, INHresistant $\mathrm{TB}$, EMB-resistant $\mathrm{TB}$, and total DR-TB dropped by $33.48,23.67,25.49,12.17 \%$, respectively, with a decrease from 20.07 to $13.35 \%, 13.49$ to $10.29 \%, 1.97$ to $1.47 \%$, respectively.

\section{Temporal trends and Joinpoint regression model}

The Joinpoint regression analysis (Table 4) revealed that total primary MR-TB rate had decreased during 20042007 (APC $=-20.6 \% \quad[95 \% \mathrm{CI}:-33.7,-4.9], Z=-3.00$, $P<0.01)$. In contrast, total primary MDR-TB rate had increased during 2004-2006 (APC $=57.9 \%$ [95\%CI: 0.3, 148.7], $Z=2.40, P<0.01)$. The rate of INH resistant TB showed a significant decline in the trend with an APC of $-2.3 \%$ during $2004-2018(P<0.01)$, while the rate of RFP resistant TB were rising significantly during 2004-2010 (APC $=16.90 \%$ [95\%CI: 5.8, 29.1], $Z=3.50, P<0.01)$.
The primary DR-TB rate among smoking and drinking subgroups had been on the rise during 2011-2018 (APC $=7.1 \%$ [95\%CI: 0.8, 13.8], $Z=2.90, P<0.01)$ and 2008-2018 (APC =6.7\% [95\%CI: 1.6,12.1], $\mathrm{Z}=3.00, P<$ 0.01 ), respectively. Of $45-64$ year-old TB group, the primary DR-TB rate decline during 2004-2007(APC = 20.5\% [95\%CI: $-33.2,-5.3$ ], $Z=-3.10, P<0.01)$. DR-TB among elderly TB cases ( $>65$ years) showed a sharply drop during 2004-2009 (APC $=-12.7 \%$ [95\%CI: -21.8 ,2.4], $Z=-2.70, P<0.01)$.

\section{Discussion}

Our study had enrolled 11,467 newly diagnosed TB cases with DST results at $36 \mathrm{~TB}$ surveillance centers across Shandong, China from 2004 to 2018 to evaluate the epidemiology and high risk factors of primary TB drug resistance, and we found that ongoing transmission of drug resistant MTB remained to be the major mechanism of DR-TB in China, approximately one fifth of new TB cases were primary DR-TB while $1.3-4.4 \%$ were MDR-TB, slightly differed from previous researches in Zhejiang, Jiangsu, Heilongjiang, China [10, 18-20]. Trends in annual prevalence of primary DR revealed that the rates of MDR-TB (from 1.32 to $3.73 \%$ ), PDR-TB (from 2.96 to $4.30 \%$ ) began to increase slowly along with that of MR-TB (from 20.07 to $13.35 \%$ ) decreasing, However, MR-TB still dominated the drug resistance rate. In a similar way the proportion of these three sub-types of DR-TB changed during our study. The increment in 
Table 3 The temporal change trend of primary drug resistance rate among newly diagnosed TB cases in Shandong, China, 2004-2018

\begin{tabular}{|c|c|c|c|c|c|c|c|c|c|c|c|c|c|c|c|c|}
\hline \multirow[t]{2}{*}{ Characteristics } & \multicolumn{15}{|c|}{ Primary drug resistance rate(\%) } & \multirow{2}{*}{$\begin{array}{l}\text { Change }^{\mathrm{a}}(\%) \\
2005-2017\end{array}$} \\
\hline & 2004 & 2005 & 2006 & 2007 & 2008 & 2009 & 2010 & 2011 & 2012 & 2013 & 2014 & 2015 & 2016 & 2017 & 2018 & \\
\hline DR-TB (Total) & 24.34 & 17.91 & 19.73 & 17.81 & 19.72 & 13.97 & 30.28 & 19.75 & 17.99 & 17.83 & 17.26 & 18.66 & 19.10 & 19.44 & 21.38 & -12.17 \\
\hline \multicolumn{17}{|l|}{ Type } \\
\hline MR-TB & 20.07 & 13.48 & 12.66 & 9.58 & 11.49 & 8.48 & 18.35 & 11.59 & 9.47 & 11.25 & 9.44 & 11.97 & 11.44 & 12.37 & 13.35 & -33.48 \\
\hline MDR-TB & 1.32 & 1.01 & 3.35 & 3.29 & 2.84 & 2.74 & 5.50 & 3.23 & 4.40 & 3.59 & 3.22 & 3.14 & 2.96 & 2.78 & 3.73 & 183.71 \\
\hline PDR-TB & 2.96 & 3.42 & 3.72 & 4.94 & 5.39 & 2.74 & 4.59 & 4.56 & 4.11 & 2.99 & 4.49 & 3.55 & 4.70 & 4.17 & 4.30 & 45.20 \\
\hline \multicolumn{17}{|l|}{ Age (years) } \\
\hline $15-44$ & 18.59 & 15.53 & 19.35 & 21.03 & 19.93 & 14.12 & 29.17 & 19.55 & 17.88 & 18.94 & 16.08 & 21.49 & 19.58 & 20.49 & 24.44 & 31.49 \\
\hline $45-64$ & 32.47 & 19.31 & 19.92 & 16.10 & 18.69 & 14.39 & 41.18 & 21.99 & 19.34 & 19.64 & 19.93 & 18.35 & 21.67 & 20.00 & 21.10 & -35.01 \\
\hline$>65$ & 28.17 & 20.30 & 19.80 & 14.72 & 20.23 & 12.22 & 10.53 & 17.05 & 16.40 & 13.77 & 15.82 & 15.11 & 15.50 & 17.77 & 17.84 & -36.66 \\
\hline \multicolumn{17}{|l|}{ Sex } \\
\hline Male & 25.91 & 17.49 & 19.21 & 18.37 & 21.11 & 15.95 & 27.84 & 20.95 & 17.91 & 18.47 & 17.95 & 18.08 & 19.93 & 19.76 & 21.60 & -16.62 \\
\hline Female & 20.24 & 19.30 & 21.29 & 16.15 & 15.85 & 8.16 & 50.00 & 13.33 & 18.52 & 13.99 & 14.10 & 21.39 & 14.91 & 17.65 & 20.27 & 0.16 \\
\hline \multicolumn{17}{|l|}{ Cavity } \\
\hline Yes & 20.83 & 12.00 & 25.27 & 18.29 & 20.73 & 10.38 & 35.29 & 18.53 & 25.39 & 18.59 & 18.34 & 19.73 & 20.70 & 20.40 & 22.13 & 6.24 \\
\hline No & 24.90 & 19.51 & 17.72 & 17.46 & 19.26 & 15.71 & 25.00 & 20.55 & 13.10 & 17.08 & 15.63 & 19.24 & 15.43 & 21.17 & 18.34 & -26.34 \\
\hline \multicolumn{17}{|l|}{ Smoking } \\
\hline Yes & & & & & 21.57 & 13.04 & & 15.00 & 15.09 & 19.23 & 18.78 & 19.48 & 16.67 & 21.05 & 26.09 & 20.95 \\
\hline No & & & & 23.08 & 15.63 & 16.67 & & 21.23 & 16.67 & 17.24 & 16.11 & 19.18 & 19.37 & 19.12 & 19.43 & -15.79 \\
\hline \multicolumn{17}{|l|}{ Drinking } \\
\hline Yes & & & & & 10.26 & 11.76 & & 18.86 & 12.39 & 19.34 & 21.34 & 19.35 & 16.45 & 15.74 & 26.55 & 158.85 \\
\hline No & & & & 25.00 & 19.29 & 16.67 & & 19.53 & 17.52 & 17.41 & 15.59 & 19.44 & 19.39 & 20.16 & 19.45 & -22.18 \\
\hline \multicolumn{17}{|l|}{ First-line drugs } \\
\hline $\mathrm{INH}$ & 13.49 & 11.07 & 12.16 & 11.62 & 12.62 & 8.98 & 16.51 & 11.21 & 12.82 & 10.46 & 8.98 & 8.82 & 9.40 & 8.84 & 10.29 & -23.67 \\
\hline RFP & 1.97 & 1.81 & 3.97 & 3.78 & 3.97 & 3.49 & 6.42 & 4.46 & 5.17 & 4.98 & 5.06 & 4.87 & 4.70 & 4.17 & 5.77 & 192.31 \\
\hline EMB & 1.97 & 0.80 & 0.99 & 1.84 & 2.13 & 0.25 & 1.83 & 2.18 & 1.34 & 0.80 & 2.42 & 1.01 & 1.53 & 1.89 & 1.47 & -25.49 \\
\hline SM & 12.17 & 9.46 & 12.41 & 13.36 & 12.91 & 8.73 & 21.10 & 12.73 & 11.48 & 11.35 & 13.12 & 13.79 & 14.61 & 14.52 & 15.27 & 25.47 \\
\hline
\end{tabular}

Primary drug resistance rate(\%) were calculated as follows: (the quantity of each DR-TB subgroups/ the quantity of corresponding primary TB cases (total) in the same year)* $100 \%$, for example, primary drug resistance rate(\%) of female TB cases in $2018=$ the quantity of female DR-TB cases in $2018 /$ the quantity of total female primary TB cases in 2018)*100\%

Joinpoint regression of these crude ratios were shown in Table 4

$T B$ Tuberculosis, $D R$-TB Drug-resistant tuberculosis, MR-TB Mono-resistant tuberculosis, MDR-TB Multi-resistant tuberculosis, $P D R-T B$ Polydrug resistant tuberculosis, $E M B$ Ethambutol, INH Isoniazid, RFP Rifampin, SM Streptomycin

${ }^{a}$ The $\%$ changes were calculated as follows: (incidence in 2018-incidence in 2005)/incidence in 2005

both MDR-TB and PDR-TB alarmed us that it would continue to be a tough challenge to $\mathrm{TB}$ elimination strategy in China $[4,21]$.

According the temporal trend in proportions of different primary DR-TB subgroups each year, we pointed out that primary resistance patterns were shifting from female, non-cavity, INH resistant TB, and MR-TB groups to male, cavity, RFP/SM resistant TB, and MDR-TB groups in Shandong China from 2004 to 2018, and more than two thirds were male or had a resistance to SM, but more than half of the present primary DR-TB cases were still MRTB. The percentage of males among MDR-TB cases were more than $70 \%$, which was consistent with previous researches in Bangladesh [22], Mozambique [23], et al., but in contrast with Ethiopia [24] and Lianyungang city, China [25] where females were in majority. The major reasons for this phenomenon were that with genuine gender or behavioral differences [25], men had a significant higher risk of contracting and dying from TB than women [26], and it was reported by WHO that TB incidence around China in 2017 for male patients (67.5\%) was twice as much as females (32.5\%) [7]. In accordance with Li D [27] and Yeom's [28] results, we found that cavities had turned to be more common for primary DR TB since 2012 in 
Table 4 Annual percentage change in primary drug-resistant rate of Mycobacterium tuberculosis in Shandong, China, 2004-2018

\begin{tabular}{|c|c|c|c|c|}
\hline Variables & Phases & APC & Test Statistic (t) & Prob $>|t|^{*}$ \\
\hline \multicolumn{5}{|l|}{ Type } \\
\hline \multirow[t]{3}{*}{ DR-TB (Total) } & 2004-2007 & $-9.2(-21.2,4.6)$ & -1.60 & 0.20 \\
\hline & 2007-2010 & $6.2(-20.1,41.1)$ & 0.50 & 0.60 \\
\hline & 2010-2018 & $-0.9(-4.0,2.2)$ & -0.70 & 0.50 \\
\hline \multirow[t]{3}{*}{ MR-TB } & 2004-2007 & $-20.6^{*}(-33.7,-4.9)$ & -3.00 & 0.00 \\
\hline & $2007-2010$ & $7.5(-25.1,54.3)$ & 0.50 & 0.60 \\
\hline & 2010-2018 & $-0.2(-4,3.8)$ & -0.10 & 0.90 \\
\hline \multirow[t]{3}{*}{ MDR-TB } & 2004-2006 & $57.9^{*}(0.3148 .7)$ & 2.40 & $P<0.001$ \\
\hline & 2006-2010 & 11.4(-11.3,39.7) & 1.10 & 0.30 \\
\hline & 2010-2018 & $-3.4(-8,1.6)$ & -1.60 & 0.10 \\
\hline \multirow[t]{3}{*}{ PDR-TB } & 2004-2007 & $16.7(-17.6,65.4)$ & 1.00 & 0.30 \\
\hline & 2007-2013 & $-3.8(-17.7,12.4)$ & -0.60 & 0.60 \\
\hline & 2013-2018 & $4.1(-10.9,21.6)$ & 0.60 & 0.60 \\
\hline \multicolumn{5}{|l|}{ Age (years) } \\
\hline \multirow[t]{3}{*}{$15-44$} & 2004-2010 & $3.8(-4.1,12.4)$ & 1.10 & 0.30 \\
\hline & 2010-2014 & $-5.3(-25.2,19.9)$ & -0.50 & 0.60 \\
\hline & 2014-2018 & $8.4(-6.6,25.8)$ & 1.30 & 0.20 \\
\hline \multirow[t]{3}{*}{$45-64$} & 2004-2007 & $-20.5^{*}(-33.2,-5.3)$ & -3.10 & $P<0.001$ \\
\hline & 2007-2010 & $18.1(-16.8,67.7)$ & 1.10 & 0.30 \\
\hline & 2010-2018 & $-3.2(-6.8,0.6)$ & -2.00 & 0.10 \\
\hline \multirow[t]{2}{*}{$>65$} & 2004-2009 & $-12.7^{*}(-21.8,-2.4)$ & -2.70 & 0.00 \\
\hline & 2009-2018 & $3.3(-1.3,8.1)$ & 1.60 & 0.10 \\
\hline \multicolumn{5}{|l|}{ Sex } \\
\hline \multirow[t]{2}{*}{ Female } & 2004-2008 & $-6.4(-39.8,45.4)$ & -0.30 & 0.70 \\
\hline & 2008-2018 & $1.2(-9.2,12.8)$ & 0.20 & 0.80 \\
\hline \multirow[t]{2}{*}{ Male } & 2004-2006 & $-10.3(-43.5,42.5)$ & -0.50 & 0.60 \\
\hline & 2006-2018 & $0.4(-2.3,3.2)$ & 0.40 & 0.70 \\
\hline \multicolumn{5}{|l|}{ Cavity } \\
\hline \multirow[t]{3}{*}{ No } & 2004-2006 & $-11.9(-55.5,74.7)$ & -0.40 & 0.70 \\
\hline & 2006-2014 & $-1.3(-9.9,8.2)$ & -0.30 & 0.70 \\
\hline & 2014-2018 & $3.5(-16.6,28.5)$ & 0.40 & 0.70 \\
\hline \multirow[t]{2}{*}{ Yes } & 2004-2010 & $3.2(-9.6,17.9)$ & 0.50 & 0.60 \\
\hline & 2010-2018 & $-0.1(-8.3,8.9)$ & 0.00 & 1.00 \\
\hline \multicolumn{5}{|l|}{ Smoking } \\
\hline No & 2008-2018 & $1.6(-0.5,3.8)$ & 1.80 & 0.10 \\
\hline \multirow[t]{2}{*}{ Yes } & 2008-2011 & $-7.2(-41,45.9)$ & -0.40 & 0.70 \\
\hline & $2011-2018$ & $7.1^{*}(0.8,13.8)$ & 2.90 & $P<0.001$ \\
\hline \multicolumn{5}{|l|}{ Drinking } \\
\hline \multirow[t]{2}{*}{ No } & 2008-2014 & $-1.2(-4.9,2.6)$ & -0.80 & 0.40 \\
\hline & 2014-2018 & $4.5(-2.7,12.1)$ & 1.60 & 0.20 \\
\hline Yes & 2008-2018 & $6.7^{*}(1.6,12.1)$ & 3.00 & $P<0.001$ \\
\hline \multicolumn{5}{|l|}{ First-line drugs } \\
\hline $\mathrm{INH}$ & 2004-2018 & $-2.3^{*}(-4.3,-0.3)$ & -2.50 & $P<0.001$ \\
\hline RFP & 2004-2010 & $16.9^{*}(5.8,29.1)$ & 3.50 & $P<0.001$ \\
\hline
\end{tabular}


Table 4 Annual percentage change in primary drug-resistant rate of Mycobacterium tuberculosis in Shandong, China, 2004-2018 (Continued)

\begin{tabular}{cllll}
\hline Variables & Phases & APC & Test Statistic $(\mathrm{t})$ & Prob $^{\prime}|\mathrm{t}|^{*}$ \\
\hline \multirow{2}{*}{ EMB } & $2010-2018$ & $-1.1(-7.3,5.4)$ & -0.40 & 0.70 \\
& $2004-2009$ & $-6.9(-30.5,24.6)$ & -0.50 & 0.60 \\
\multirow{2}{*}{ SM } & $2009-2018$ & $6(-5.9,19.4)$ & 1.10 & 0.30 \\
\hline
\end{tabular}

APC Annual percent change, TB Tuberculosis, DR-TB Drug-resistant tuberculosis, MR-TB Mono-resistant tuberculosis, MDR-TB Multi-resistant tuberculosis, PDR-TB Polydrug resistant tuberculosis, EMB Ethambutol, INH Isoniazid, RFP Rifampin, SM Streptomycin

${ }^{*} P<0.05$

China. Low effectiveness of anti-TB drug sensitivity towards primary DR-TB, limited drug penetration into cavities, specific microorganism virulence and immune status of patients may contribute to this phenomenon [27-29]. However, another reason the more important may be the imbalance between compensatory evolution and compensation cost of drug resistant MTB strains [30, 31]. Four out of ten primary DR-TB cases were in the age group of $15-44$ years, followed by groups of $45-64$ years $(33.5 \%)$ and $>65$ years (22.37\%), suggesting that DR-TB prevention and control should focus more on young and middle aged patients. In recent years, TB patients who were alcohol users and smokers were associated with a higher annual primary DR-TB prevalence than those who were nonsmokers and non-drinkers. However, when total TB cases across 2004-2018 were involved in bivariate analysis, personal behaviours like smoking and alcohol use were no longer predictors of primary DR-TB on bivariate analysis, in contrast to findings in India [32] and Botswana [33] where alcohol use was a risk factor for MDR-TB, but consistent with studies in Nepal [34], indicating that it may be distinguished by regions and phases.

Directly observed treatment, short-course (DOTS) strategy had been put forward in China since 1990s, and was expanded to cover all smear-positive and smearnegative TB patients since 2005 instead of previous policy that only patients diagnosed with smear-positive or severe smear-negative TB were available to free treatment $[35,36]$. As a consequence, TB prevalence has declined sharply from 1990 to 2010 [36, 37], in accordance with which both the percentage of primary DR-TB, RFP resistant TB and the drug resistant rate of 45-64 year-old, $>65$ year-old or female group dropped significantly during 2004-2007. Possible reason for this trend was the reduction of acquired DR-TB due to the free treatment policy in China (35-37), in other words, the source of primary DR-TB had been controlled effectively. An obvious increment in primary MDR-TB and RFP resistant TB during 2004-2008 had been observed, and one hypothesis was that MDR/RR-TB were more difficult to treat than drug-susceptible $\mathrm{TB}$ and other types of DR-TB, thus it was hard to prevent the newly transmission of previous MDR/RR-TB as well $[4,7,38]$.
Furthermore, evidence has shown that substantial costs associated with TB diagnosis and treatment remained a heavy financial burden for TB patients in spite of the "free" TB care policy [35]. Other mechanisms remained to be further explored. Another issue of concern was that people with smoking or drinking habits tended to be more susceptible to infection of drug-resistant MTB $[39,40]$, consistent to our research since 2008. In comparison with never-smokers, current smokers had an excess risk of pulmonary TB (adjusted HR, 2.87; 95\% CI, $2.00-4.11 ; p<0.001)[40]$.

Our study had several advantages. First, our study covered all DST data of Shandong province from 2004 to 2018, which is the second largest province in China with a population of nearly 90 million, thus the findings of our research were more likely to be rolled out nationally. Second, we distinguished primary DR-TB from acquired $\mathrm{DR}-\mathrm{TB}$, and the total TB or DR-TB population were stratified by various factors including sex, age, smoking history, drinking history, cavity and so on, other than most previous epidemiological researches in DR-TB that considered new and relapse DR-TB together $[1,2,9,10]$. Third, we proposed a Joinpoint regression model to describe the temporal trend and turning point of TB control in Shandong, China.

There were also some limitations of our study. First, drug sensitive tests were not routinely carried out among TB cases, thus our data from TB monitoring stations were affected by screening intensity and local medical conditions, and could be overestimated due to selection bias. Second, diversities in technical levels and experimental conditions in different TB monitoring stations may contributed to unavoidable bias. Third, DST for second-line anti-TB drugs were seldom performed by TB monitoring stations in Shandong, China, thus the epidemiology of primary resistance to second-line anti$\mathrm{TB}$ drugs and extensive drug-resistant $\mathrm{TB}$ (XDR-TB) could not be estimated.

\section{Conclusion}

Our study had described the epidemiological features and temporal trend of primary DR-TB in Shandong province, China from 2004 to 2018 to evaluate the 
current situation of TB prevention and control. We found that the proportion of primary DR-TB and MRTB had reduced by more than $12 \%$ since 2004 , and were $21.38,13.35 \%$ in 2018 respectively. Moreover, primary drug resistance patterns were shifting from female, noncavity, INH resistant TB, and MR-TB groups to male, cavity, RFP/SM resistant TB, and MDR-TB groups, even more than two thirds were male or had a resistance to $\mathrm{SM}$, but more than half of the present primary DR-TB cases were still MR-TB. This study indicates the DOTS strategy and "free" policy in China had achieved some effects in TB control, but considering the increment of drug resistance rate among some special population, more attention should be focused on female, cavity, smoking, drinking, 15 to 44 year-old TB subgroups. In addition, more project support, high-quality training for medical staff and enhanced public awareness of TB prevention and control are also necessary for $\mathrm{TB}$ elimination goal in China.

\section{Appendix}

Table $\mathbf{5}$ Changes in proportions of different primary drugresistant mycobacterium tuberculosis subgroups, Shandong, China, 2004-2018 a

\begin{tabular}{llllll}
\hline Subgroups & $X^{2}$ & $P$ value & $R^{2}$ & $X$-coefficient & SE \\
\hline MR-TB (\%) & 6.287 & 0.012 & 0.1780 & -0.0073 & 0.6852 \\
MDR-TB (\%) & 5.376 & 0.020 & 0.2034 & 0.0051 & 0.1221 \\
PDR-TB (\%) & 0.933 & 0.334 & 0.0505 & 0.0022 & 0.1927 \\
15-44 years(\%) & 0.673 & 0.412 & 0.0822 & -0.0026 & 0.4504 \\
45-64 years(\%) & 2.837 & 0.092 & 0.2046 & 0.0045 & 0.3077 \\
>65 years(\%) & 0.506 & 0.477 & 0.0399 & -0.0020 & 0.2402 \\
Male (\%) & 12.570 & $P<0.001$ & 0.4834 & 0.0081 & 0.7595 \\
Female (\%) & 12.570 & $P<0.001$ & 0.4834 & -0.0081 & 0.2405 \\
Cavity (\%) & 120.530 & $P<0.001$ & 0.7022 & 0.0255 & 0.1202 \\
Non-cavity (\%) & 120.530 & $P<0.001$ & 0.7244 & -0.3673 & 0.8358 \\
Smoking (\%) & 0.392 & 0.531 & 0.6400 & 0.0179 & -0.0268 \\
Non-smoking (\%) & 0.392 & 0.531 & 0.7506 & 0.0551 & -0.0996 \\
Drinking (\%) & 0.194 & 0.659 & 0.5352 & 0.0147 & -0.0229 \\
Non-drinking (\%) & 0.194 & 0.659 & 0.7634 & 0.0571 & -0.1006 \\
INH (\%) & 14.725 & $P<0.001$ & 0.4269 & -0.0112 & 0.6600 \\
RFP (\%) & 16.785 & $P<0.001$ & 0.5355 & 0.0101 & 0.1416 \\
EMB (\%) & 0.746 & 0.388 & 0.0300 & 0.0012 & 0.0659 \\
SM (\%) & 22.076 & $P<0.001$ & 0.5365 & 0.0133 & 0.5620 \\
\hline NH (\%), & $1 \%$ (\%), EMB &
\end{tabular}

INH (\%), RFP (\%), EMB (\%), and SM (\%) refer to the proportion of primary INH/ $\mathrm{RFP} / \mathrm{EMB} / \mathrm{SM}$ resistant TB among total primary DR TB cases, respectively ${ }^{a} E M B$ Ethambutol, INH Isoniazid, RIF Rifampin, SM Streptomycin, TB Tuberculosis

MR-TB Mono-resistant tuberculosis, MDR-TB Multi-resistant tuberculosis, PDR-TB Polydrug resistant tuberculosis, EMB Ethambutol, INH Isoniazid, RFP Rifampin, SM Streptomycin

\section{Abbreviations}

AAPCs: Average annual percent changes; APCs: Annual percent changes; Cl: Confidence interval; DR-TB: Drug-resistant tuberculosis; DST: Drug susceptibility test; EMB: Ethambutol; INH: Isoniazid; MDR-TB: Multi-resistant tuberculosis; MR-TB: Mono-resistant tuberculosis; MTB: Mycobacterium tuberculosis; ORs: Odds ratios; PDR-TB: Polydrug resistant tuberculosis; RFP: Rifampin; RR-TB: Rifampin-resistant tuberculosis; SM: Treptomycin; TB: Tuberculosis; WGS: Whole genome sequencing; WHO: World health organization; XDR-TB: Extensive drug resistant TB

\section{Acknowledgments}

We thank Shandong Provincial Hospital, Shandong Provincial Chest Hospital, 13 municipal-level and 21 county-level local health departments for drug susceptibility data, sociodemographic and clinical data.

\section{Authors' contributions}

$\mathrm{HCL}$ and YFL conceived and designed the study. HCL, CBY, LLC, and LG directed its implementation including the data analysis and writing of the paper. WMS and YFL analyzed the data; YL, QYZ, JYL, TTX, SJL and NNT contributed materials/analytic tools; WMS, YFL and HCL wrote and revised the manuscript. All authors reviewed and approved the manuscript.

\section{Funding}

This work was supported by Department of Science \& Technology of Shandong Province (CN)(No.2007GG30002033; No.2017GSF218052) and Jinan Science and Technology Bureau (CN) (No.201704100).

Availability of data and materials

Data can be available through contact with the corresponding author.

\section{Ethics approval and consent to participate}

The protocols applied in this study were approved by the Ethics Committee of Shandong Provincial Hospital, affiliated with Shandong University (SPH) and and the Ethic Committee of Shandong Provincial Chest Hospital (SPCH), China. Before analysis, patient records were anonymized and deidentified.

\section{Consent for publication}

Not applicable.

\section{Competing interests}

The authors declare that they have no competing interests.

\section{Author details}

'Department of Respiratory Medicine, Shandong Provincial Hospital Affiliated to Shandong University, Jinan 250021 , Shandong, China. ${ }^{2}$ Cheeloo College of Medicine, Shandong University, Jinan 250012, Shandong, China. ${ }^{3}$ School of Medicine and Life Sciences, University of Jinan-Shandong Academy of Medical Sciences, Jinan 250200, Shandong, China. ${ }^{4}$ Peking Union Medical College, Beijing 100005, China. ${ }^{5}$ Department of Respiratory Medicine, Shandong Provincial Chest Hospital, Jinan 250013, Shandong, China. ${ }^{6}$ NHC Key Laboratory of Systems Biology of Pathogens, Institute of Pathogen Biology, and Center for Tuberculosis Research, Chinese Academy of Medical Sciences and Peking Union Medical College, Beijing 100730, China.

${ }^{7}$ Department of Biostatistics, School of Public Health, Shandong University, Jinan 250012, Shandong, China. ${ }^{8}$ Jinan Municipal Center for Disease Control and Prevention, Jinan 250021, Shandong, China. ${ }^{9}$ Shandong University of Traditional Chinese Medicine, Jinan 250355, Shandong, China.

Received: 23 May 2019 Accepted: 25 September 2019

Published online: 18 October 2019

References

1. Gandhi NR, Nunn P, Dheda K, Schaaf HS, Zignol M, van Soolingen D, et al. Multidrug-resistant and extensively drug-resistant tuberculosis: a threat to global control of tuberculosis. Lancet. 2010;375(9728):1830-43.

2. Yang Y, Zhou C, Shi L, Meng H, Yan H. Prevalence and characterization of drug-resistant tuberculosis in a local hospital of Northeast China. Int J Infect Dis. 2014;22:83-6.

3. World Health Organization. Antimicrobial Resistance. Geneva: World Health Organization; 2018. http://www.who.int/newsroom/fact-sheets/detail/ antimicrobial-resistance 
4. Zhao Y, Zhao B, Xu S, Wang S, Wang L, Wang Y, et al. National survey of drug-resistant tuberculosis in China. N Engl J Med. 2012;366(23):2161-70.

5. Yang C, Luo T, Shen X, Wu J, Gan M, Xu P, et al. Transmission of multidrugresistant Mycobacterium tuberculosis in Shanghai, China: a retrospective observational study using whole-genome sequencing and epidemiological investigation. Lancet Infect Dis. 2017;17(3):275-84.

6. Marais BJ, Sintchenko V. Epidemic spread of multidrug-resistant tuberculosis in China. Lancet Infect Dis. 2017;17(3):238-9.

7. World Health Organization. Global tuberculosis report 2018. Geneva: World Health Organization; 2018. http://www.who.int/tb/publications/global_report/en

8. WHO 2019. Tuberculosis in China. http://www.wpro.who.int/china/ mediacentre/factsheets/tuberculosis/en/.

9. He X, Zhang X, Zhao J, Liu Y, Yu C, Yang G, et al. Epidemiological trends of drug-resistant tuberculosis in China from 2007 to 2014 a retrospective study. Medicine. 2016;95(15):e3336.

10. Yang C, Gao Q. Recent transmission of mycobacterium tuberculosis in China: the implication of molecular epidemiology for tuberculosis control. Front Med. 2018;12(1):76-83.

11. El Amin NM, Hanson HS, Pettersson B, Petrini B, Von Stedingk LV. Identification of non-tuberculous mycobacteria: 165 rRNA gene sequenceanalysis vs. conventional methods. Scand J Infect Dis. 2000;32:47-50.

12. Jing $H$, Wang $H$, Wang $Y$, Deng $Y$, Li $X$, Liu Z, et al. Prevalence of nontuberculous mycobacteria infection, China, 2004-2009. Emerging Infect Dis. 2012;18(3):527-8.

13. Van Rie A, Warren R, Richardson M, Gie RP, Enarson DA, Beyers N, et al. Classification of drug-resistant tuberculosis in an epidemic area. Lancet. 2000;356(9223):22-5.

14. Falzon D, Schünemann HJ, Harausz E, González-Angulo L, Lienhardt C,

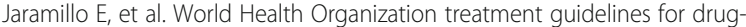
resistant tuberculosis, 2016 update. Eur Respir J. 2017:49(3):1602308.

15. Nistal-Nuño B. Joinpoint regression analysis to evaluate traffic public health policies by national temporal trends from 2000 to 2015. Int J Inj Control Saf Promot. 2018;25(2):128-33.

16. Bandi $P$, Silver D, Mijanovich T, Macinko J. Temporal trends in motor vehicle fatalities in the United States, 1968 to 2010 - a joinpoint regression analysis. Inj Epidemiol. 2015;2(1):1-11.

17. Brito AL, Monteiro LD, Ramos Junior AN, Heukelbach J, Alencar CH. Temporal trends of leprosy in a Brazilian state capital in Northeast Brazil: epidemiology and analysis by joinpoints, 2001 to 2012. Braz J Epidemiol. 2016;19(1):194

18. Wang X, Fu Q, Li Z, Chen S, Liu Z, Nelson H, et al. Drug-resistant tuberculosis in Zhejiang province, China, 1999-2008. Emerging Infect Dis. 2012;18(3):496-8.

19. Li D, Wang J, Ji B, Cui J, Pan X, Fan C, et al. Persistently high prevalence of primary resistance and multidrug resistance of tuberculosis in Heilongjiang Province, China. BMC Infect Dis. 2016;16(1):516.

20. Li X, Lu W, Zu R, Zhu L, Yang H, Chen C, et al. Comparing risk factors for primary multidrug-resistant tuberculosis and primary drug-susceptible tuberculosis in Jiangsu Province, China: a matched-pairs case-control study. Am J Trop Med Hyg. 2015;92(2):280-5

21. Raviglione M, Sulis G. Tuberculosis 2015: burden, challenges and strategy for control and elimination. Infect Dis Rep. 2016;8(2):33-7.

22. Karmaker H, Basar MA, Karim MR, et al. An epidemiological study of drug resistant tuberculosis cases: survey in the northern part of Bangladesh. Public Health Res. 2016;6(2):52-8.

23. Samo Gudo P, Cuna Z, Coelho E, Maungate S, Borroni E, Miotto $P$, et al. Is multidrug-resistant tuberculosis on the rise in Mozambique? Results of a national drug resistance survey. Eur Respir J. 2011;38(1):222-4.

24. Mulisa G, Workneh T, Hordofa N, Suaudi M, Abebe G, Jarso G. Multidrugresistant mycobacterium tuberculosis and associated risk factors in Oromia region of Ethiopia. Int J Infect Dis. 2015;39(C):57-61.

25. Liu Q, Zhu L, Shao Y, Song H, Li G, Zhou Y, et al. Rates and risk factors for drug resistance tuberculosis in Northeastern China. BMC Public Health. 2013;13(1):1171

26. Global tuberculosis report 2017. Geneva: World Health Organization; 2017. http://apps.who.int/medicinedocs/en/m/abstract/Js23360en/

27. Li D, He W, Chen B, Lv P. Primary multidrug-resistant tuberculosis versus drug-sensitive tuberculosis in non-HIV-infected patients: comparisons of CT findings. PLoS One. 2017;12:e0176354.
28. Yeom JA, Jeong YJ, Jeon D, Kim Kl, Kim CW, Park HK, et al. Imaging findings of primary multidrug-resistant tuberculosis: a comparison with findings of drug-sensitive tuberculosis. J Comput Assist Tomogr. 2009;33(6):956-60.

29. Fishman JE, Sais GJ, Schwartz DS, Otten J. Radiographic findings and patterns in multidrug-resistant tuberculosis. J Thorac Imaging. 1998; 13(1):65-71.

30. Brandis G, Wrande M, Liljas L, Hughes D, Medicinska fakulteten, Medicinska och farmaceutiska vetenskapsområdet, et al. Fitnesscompensatory mutations in rifampicin-resistant RNA polymerase. Mol Microbiol. 2012;85(1):142-51.

31. Cohen KA, Abeel T, Manson McGuire A, Desjardins CA, Munsamy V, Shea TP, et al. Evolution of extensively drug-resistant tuberculosis over four decades: whole genome sequencing and dating analysis of mycobacterium tuberculosis isolates from KwaZulu-Natal. PLoS Med. 2015;12(9):e1001880.

32. Zetola NM, Modongo C, Kip EC, Gross R, Bisson GP, Collman RG. Alcohol use and abuse among patients with multidrug-resistant tuberculosis in Botswana. Int J Tuberc Lung Dis. 2012:16(11):1529-34.

33. Pradhan $\mathrm{N}$ et al. Patterns of TB Drug-Resistance in a tertiary care facility in Pune, India. Clinical Microbiology: Open Access. 2013. https://doi.org/10. 4172/2327-5073.1000123

34. Marahatta SB, Kaewkungwal J, Ramasoota P, Singhasivanon P. Risk factors of multidrug resistant tuberculosis in central Nepal: a pilot study. Kathmandu Univ Med J (KUMJ). 2012;8(4):392-7

35. Jia X, Chen J, Zhang S, Dai B, Long Q, Tang S. Implementing a "free" tuberculosis (TB) care policy under the integrated model in Jiangsu, China: practices and costs in the real world. Infect Dis Poverty. 2016:5(1):1.

36. Wang $L$, Zhang $H$, Ruan $Y$, Chin DP, Xia Y, Cheng S, et al. Tuberculosis prevalence in China, 1990-2010; a longitudinal analysis of national survey data. Lancet. 2014;383(9934):2057-64.

37. Wang L, Liu J, Chin DP. Progress in tuberculosis control and the evolving public-health system in China. Lancet. 2007;369(9562):691-6.

38. Ricks PM, Mavhunga F, Modi S, Indongo R, Zezai A, Lambert LA, et al. Characteristics of multidrug-resistant tuberculosis in Namibia. BMC Infect Dis. 2012;12(1):385

39. Leung CC, Li T, Lam TH, Yew WW, Law WS, Tam CM, et al. Smoking and tuberculosis among the elderly in Hong Kong. Am J Respir Crit Care Med. 2004;170(9):1027-33.

40. Mesfin EA, Beyene D, Tesfaye A, Admasu A, Addise D, Amare M, et al. Drugresistance patterns of mycobacterium tuberculosis strains and associated risk factors among multi drug-resistant tuberculosis suspected patients from Ethiopia. PLoS One. 2018;13(6):e0197737.

\section{Publisher's Note}

Springer Nature remains neutral with regard to jurisdictional claims in published maps and institutional affiliations.

\section{Ready to submit your research? Choose BMC and benefit from:}

- fast, convenient online submission

- thorough peer review by experienced researchers in your field

- rapid publication on acceptance

- support for research data, including large and complex data types

- gold Open Access which fosters wider collaboration and increased citations

- maximum visibility for your research: over $100 \mathrm{M}$ website views per year

At BMC, research is always in progress.

Learn more biomedcentral.com/submissions 